\title{
Olfactory Bulbectomy in Infant Rats: Survival, Growth and Ingestive Behaviors ${ }^{1}$
}

\author{
DAVID L. HILL \\ Department of Oral Biology, School of Dentistry \\ University of Michigan, Ann Arbor, MI 48109 \\ AND \\ C. ROBERT ALMLI \\ Washington University School of Medicine, Programs in Occupational Therapy and Neural Sciences \\ Departments of Anatomy and Neurobiology, \\ Psychology and Preventive Medicine, St. Louis, MO 63110
}

\author{
Received 11 August 1981
}

\begin{abstract}
HILL, D. L. AND C. R. ALMLI. Olfactory bulbectomy in infant rats: Survival, growth and ingestive behaviors. PHYSIOL. BEHAV. 27(5) 811-817, 1981.-Growth and ingestive behaviors were measured for rats sustaining bilateral olfactory bulbectomies at 1 or 10 days of age. Tests designed to examine nipple-attachment capabilities were accomplished before weaning, and tests designed to examine drinking (e.g., water-deprivation), feeding (e.g., amphetamine-anorexia), and taste-related behaviors (e.g., quinine finickiness) were conducted from 35-150 days of age. Pups bulbectomized at 1 day of age displayed less severe nipple-attachment deficits than pups bulbectomized at 10 days of age; however, body weights of both bulbectomized groups were significantly below that of controls from the first day postsurgery through 150 days of age. Bulbectomized rats differed from controls on some drinking, feeding, and taste-related measures; deficits occurred most consistently on taste-related measures. The results are discussed in terms of neural and behavioral plasticity of developing animals sustaining neural damage.
\end{abstract}

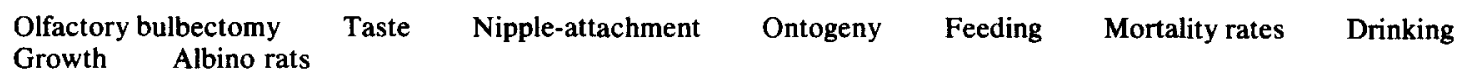

OLFACTORY cues are important for normal ingestive behaviors in adult rats (e.g., $[1,10,21])$, and current research indicates that olfactory cues are important for guiding preweaning rats to the dams' nipples $[7,8,9]$. Removal of olfactory cues from on and around the dams' nipples $[29,30]$ or damage to olfactory structures in previsual rats $[27,28,31]$ severely disrupts nipple-attachment behaviors. These nipple-attachment deficits may be reflected in the high mortality rates reported for previsual rats sustaining bilateral olfactory bulbectomy $[20,23,25,27,28,31]$.

Experiments 1 and 2 of the present investigation compared the effects of bilateral olfactory bulbectomy sustained by rats at 1 or 10 days of age on survival rates, growth, and nipple-attachment behaviors. Survivors of the bulbectomies were tested through 150 days of age to determine the presence or absence of residual deficits in feeding, drinking, and taste-related behaviors.

\section{EXPERIMENT 1}

Experiment 1 evaluated long-term survival rates, growth, and ingestive behaviors of rats sustaining bilateral olfactory bulbectomy at 1 or 10 days of age.

\section{METHOD}

Animals

One hundred twenty-two pups delivered by 16 SpragueDawley rats (Charles River "CD" strain; 7-8 pups/litter) served as subjects. Litters were housed with their dam in plastic group cages $(48.4 \times 38.1 \times 12.3 \mathrm{~cm})$ in a temperature controlled room $\left(23-27^{\circ} \mathrm{C}\right)$ under continuous illumination $[3$, $4,22,33]$. Purina rat pellets and tap water were freely available in the litter cage. Body weights for all pups were measured daily from the day of birth through the day of death or sacrifice at 150 days of age.

\section{Surgery}

Forty-two pups (males $=24$, females $=18$ ) had olfactory bulbs removed bilaterally at 1 day of age $(24 \pm 12 \mathrm{hrs}$ postpartum) and 18 pups (males $=10$, females $=8$ ) had surgi-

${ }^{1}$ This research was conducted while the authors were at Ohio University, Department of Psychology, Athens, OH 45701, and was supported by O.U.R.C. Grant No. 520 to C. Robert Almli. The manuscript was prepared while D.L.H. was supported by postdoctoral Grant No. NS06423. Address reprint requests to C. Robert Almli, Washington University School of Medicine, Department of Anatomy and Neurobiol-
ogy, 660 S. Euclid Ave., St. Louis, MO 63110 . 
cal control procedures. All littermates were removed from their dam before surgery and placed on a heating pad in a group cage. Pups at 1 day of age were immobilized by cooling them to approximately $8-11^{\circ} \mathrm{C}$ (thoracic skin temperature) in a freezer. The pups remained immobile throughout the surgical procedures which were conducted at room temperature. Following a skin incision over the skull. one point of a sharp forceps was used to penetrate the midsagittal suture between frontal bones. Both frontal bones then were reflected laterally thus exposing the dorsal surface of the olfactory bulbs. Olfactory bulbs were bilaterally aspirated through a glass pipette which was connected to a vacuum system; the vacuum was adjusted such that a controlled removal of the bulbs was accomplished. Care was taken to scrape and suction the cribiform plate of residual neural tissue, and invasion of the frontal pole was acceptable to insure complete bulbectomy. In nearly all cases blood did not enter the resultant cavity. The cavity was then completely and tightly packed with Gelfoam, the skull flap was folded back to the original position and the skin was sutured. Pups were returned to the heating pad after a bacteriostatic agent (Zephiran chloride, 1:750 parts water) was applied to the incision. The time elapsing from removal of pups from the freezer to their return to the heating pad was less than 10 min. All littermates were returned to their dam after each member of the litter had surgery and became active. Surgical control pups (at least two pups/litter) underwent the same surgical procedures as bulbectomized pups with the exception of skull opening and olfactory bulb removal.

Sixty-two additional pups were either bulbectomized at 10 days of age (males $=24$, females $=20$ ) or had surgical control procedures (males $=9$, females $=9$ ). Procedures were the same as described for 1-day-old bulbectomized or control pups except diethyl ether was used as the anesthetic agent and burr holes were drilled in the skull to expose the entire dorsal surface of the olfactory bulbs.

\section{Procedures}

All rats bulbectomized at 1 day of age were housed with their littermates and dam through 30 days of age; thereafter, they were housed individually in rack-mounted cages $(24.1 \times 17.8 \times 19.0 \mathrm{~cm})$ with access to Purina rat pellets and water through 150 days of age. Twelve rats bulbectomized at 10 days of age were housed with their littermates and dam and all $12(100 \%)$ of these bulbectomized rats died within 6 days postsurgery. None of these rats had milk in their stomachs at death, and it was assumed that the rats died of starvation. Because of the absolute mortality rate of 10-day-old bulbectomized rats housed with littermates and dam, the remaining 32 10-day-old bulbectomized rats were separated from the dam at 2-3 days postsurgery and housed in group cages (up to 4 pups/cage) with access to fresh, highly palatable and moist diets. Additional heat was applied to these group cages by a heating pad, and special diets were chocolate chip cookies soaked in Similac infant formula and Gerber Hi-Protein infant cereal liquified with Similac infant formula. The special diets were replaced each $6 \mathrm{hr}$ and the diets were available to the pups in petri dishes. From 30 through 150 days of age, the 10-day-old bulbectomized rats were housed individually in rack-mounted cages with access to Purina rat pellets and water.

Daily water intakes $(\mathrm{ml})$ were measured from 35-150 days of age. Responses to the following battery of ingestive tests were conducted from 35-150 days of age with the order of test presentation varied between rats and with at least "days elapsing between test days. These tests have been described in detail elsewhere $[3,4,22,24]$.

Drinking lests. Three-hour water intakes were measured following SC injections of $16 \% \mathrm{NaCl}$ solution $(1.0 \mathrm{ml} / 300 \mathrm{~g}$ body weight) and after 24-hr water deprivation. Water in takes were also measured during $24 \mathrm{hrs}$ of food deprivation. No food was available during testing.

Fe'ding tests. Two-hour food intakes were measured after 24-hr food deprivation and after IP injections of D-amphetamine sulfate solution $(1.5$ and $3.0 \mathrm{mg} / \mathrm{kg}$ body weight; $1.5 \mathrm{mg} / \mathrm{ml}$ ) or a control injection of physiological saline. Rats were $24-\mathrm{hr}$ food deprived prior to the amphetamine test. Water was available during the feeding tests.

Taste tests. Taste finickiness was evaluated by recording fluid intakes of $0.01 \%$ and $0.05 \%$ quinine hydrochloride solutions for two separate 24-hr periods; each solution was the only fluid source available. Twenty-four hour intakes of a saline solution $(0.1 \% \mathrm{NaCl})$ and tap water were measured in a two-bottle preference test. Food was freely available during the taste test.

\section{Post-Mortem Examination}

Rats surviving to 150 days of age were sacrificed with an overdose of diethyl ether and perfused with $0.87 \%$ physiological saline and $10 \%$ Formalin solutions. Rats that died prior to 150 days of age had the superficial tissue removed from around their skulls and the skulls were soaked in $10 \%$ Formalin solution for at least 2 weeks. The olfactory bulb cavity was carefully dissected to determine the completeness of the bulbectomy, and the olfactory bulb cavity was inspected under a dissecting microscope to determine the presence or absence or residual olfactory bulb tissue. Rats included in this study were those that sustained unambigious complete removal of the olfactory bulbs bilaterally

\section{RESULTS}

Daily body weights and daily water intakes were analyzed by analysis of variance with posttest comparisons accomplished with Tukey's [32] HSD procedure for main effects and Cicchetti's [11] test for significant interactions. Ingestive test (drinking, feeding, and taste) data were analyzed by two-tailed $t$-tests.

\section{Post-Mortem Examination}

The completeness of the bilateral olfactory bulbectomy sustained by the rats of the present study is evidenced in the photographs presented in Fig. 1. Presented are representative brains of male OB-1, OB-10, and Day-1 control rats sacrificed at 150 days of age. Careful examination of the brains and bulb cavity during dissection revealed that the olfactory bulb cavity had been cleared of almost all tissue and no organized olfactory bulb material remained. The completeness of bilateral olfactory bulbectomy was also indicated by the absence of the lateral olfactory tracts and the damage inflicted to the anterior frontal pole region which was sustained while clearing the cavity of caudal olfactory bulb tissue during surgery. Bulbectomies in both OB-1 and OB-10 rats were equally complete, as were the bulbectomies between rats that presumably starved to death versus those that survived to 150 days of age.

In some rats, the bulb cavity had apparently been incompletely packed with Gelfoam and the frontal pole had her- 


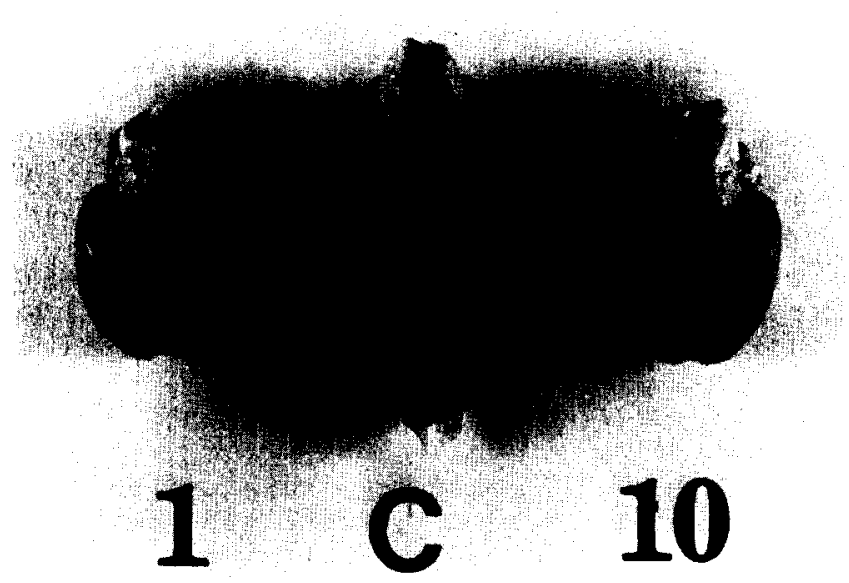

FIG. 1. Photographs of the brains of rats sustaining bilateral olfactory bulbectomy at 1 or 10 days of age. The control rat (c) was from the Day- 1 group. All rats were males and were sacrificed at 150 days of age. Note the lateral olfactory tracts (light fiber bands) in the control brain and the absence of the lateral olfactory tracts in the OB-1 and OB-10 brains.

niated into the bulb cavity. The difficulty in determining the completeness of those bulbectomies resulted in the exclusion of those rats from the present study. For those rats, the entire brain narrowed and elongated.

Survival rates and body weight. Of the 42 rats sustaining olfactory bulbectomy at 1 day of age (OB-1), 7 died by 5 days of age (17\% of total), 8 more died by 10 days of age ( $36 \%$ of total) and 2 more died by 15 days of age ( $40 \%$ of total). The remaining 25 OB-1 rats ( $60 \%$ of total) survived through the day of sacrifice at 150 days of age.

The two groups of rats bulbectomized at 10 days of age (OB-10) had very different survival rates. Of the $12 \mathrm{OB}-10$ rats that remained with the dam, $100 \%$ had died by 6 days postsurgery. For the 32 rats that were early-weaned with the special diets, 2 died by 5 days postsurgery and 8 more died by 10 days postsurgery ( $32 \%$ of total). The remaining 22 OB- 10 rats (68\% of total) survived through sacrifice at 150 days of age. It is possible that even higher survival rates for $\mathrm{OB}-10$ rats may have been achieved if the special diets would have been added to the cages containing the bulbectomized rats and their dam.

Presented in Fig. 2 are the mean body weights (expressed as a percentage of the corresponding mean body weights of control rats) of male and female OB-1 and OB-10 rats on the day of surgery, 9 days postsurgery, and at 20-150 days of age. The OB-10 rats included in this figure were those that were early-weaned to special diets. No body weight differences existed between same-sexed control groups (Day-1 and Day-10) at 30 days of age so their data were combined $(p>0.05)$.

Male and female OB-1 rats were at control levels for body weight on the day of surgery (1 day of age). Immediately postsurgery, these rats lost body weight; however, all OB-1 rats had gained body weight by the end of the second day postsurgery. The mean number of days for OB-1 rats to reach or exceed their prelesion body weights was 3.2 days
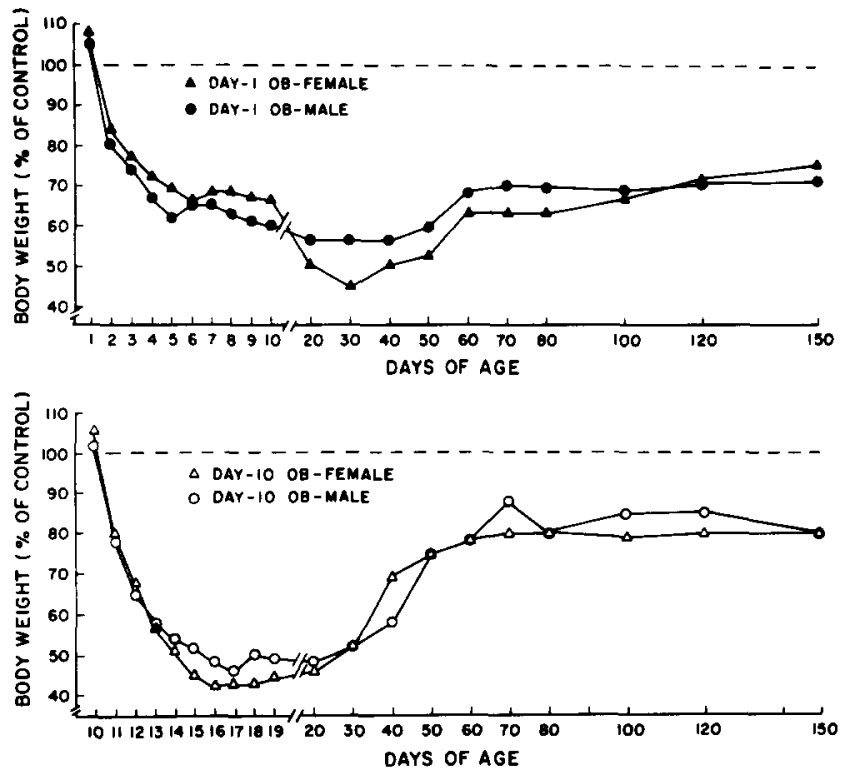

FIG. 2. Mean body weights for male (circles) and female (triangles) rats bulbectomized at 1 day of age (top) or 10 days of age (bottom) from the day of surgery through 150 days of age. Weights are expressed as a percentage of control weights.

( $\min =2$ days; $\max =5$ days). Although male and female OB-1 rats gained body weight soon after bulbectomy, they did so at a slower rate than control rats through approximately 20 days of age. At 20 days of age, OB-1 rats were $50-58 \%$ of control body weights. From approximately 30-60 days of age, male and female OB-1 rats gained body weight at a faster rate than did controls, yet, the OB-1 rats remained approximately $70 \%$ of control body weights from $60-150$ days of age (see Fig. 2).

Male and female OB-10 rats were at control levels for body weight on the day of surgery (10 days of age). Postsurgery, the OB-10 rats lost body weight through the fifth day post surgery. The mean number of days to reach or exceed their prelesion body weight for OB-10 rats was 13.4 days ( $\min =6$ days; $\max =33$ days). Incidentally, the fifth day postsurgery ( 15 days of age) was the day that all OB-10 rats that eventually survived to 150 days of age had begun to eat the special diets. Once ingesting the special diets, OB-10 rats were able to stabilize and then increase their body weights relative to control body weights. OB-10 rats' body weights stabilized at approximately 16 days of age (43-48\% of control weights), and they gained body weight at a faster rate than controls from 20-60 days of age. All OB-10 rats were eating lab chow exclusively by 30 days of age, thus, they continued to gain relatively more weight than controls while on the same diet. Male and female OB-10 rats remained approximately $80-85 \%$ of control weights from $60-150$ days of age.

Statistical comparisons revealed that OB-1 and OB-10 rats weighed reliably less than controls by $24 \mathrm{hrs}$ postsurgery and they remained significantly below control body weights through 10 and 20 days of age, respectively (OB-1-F $(9,387)$ $=45.47$; OB-10-F $(10,290)=128.1$; posttests $p<0.01)$. Furthermore, male and female OB-1 and OB-10 rats weighed reliably less than their respective control groups through 150 
TABLE 1

RESULTS OF DRINKING, TASTE, AND FEEDING TESTS FOR RATS SUSTAINING BILATERAL OLFACTORY BULBECTOMY AT I (OB-1) OR 10 (OB-10) DAYS OF AGE

\begin{tabular}{|c|c|c|c|c|}
\hline & \multicolumn{2}{|c|}{ OB-1 } & \multicolumn{2}{|c|}{ OB- 10} \\
\hline & Male & Female & Male & Female \\
\hline \multicolumn{5}{|l|}{ Drinking Tests } \\
\hline Cellular Dehydration & $101 \%$ & $109 \%$ & $115 \%$ & $142 \% *$ \\
\hline 24 hr Food Deprivation & 86 & 76 & 136 & 78 \\
\hline $24 \mathrm{hr}$ Water Deprivation & 94 & $70^{*}$ & 89 & 119 \\
\hline \multicolumn{5}{|l|}{ Taste Tests } \\
\hline \multicolumn{5}{|l|}{ Low Quinine } \\
\hline$(0.01 \%)$ & 128 & $37^{*}$ & $26^{*}$ & $45^{*}$ \\
\hline \multicolumn{5}{|l|}{ High Quinine } \\
\hline$(0.05 \%)$ & 135 & $33^{*}$ & $47^{*}$ & $46^{*}$ \\
\hline \multicolumn{5}{|l|}{$\mathrm{NaCl}$ Preference } \\
\hline$\left(\mathrm{ml} \mathrm{NaCl} / \mathrm{ml} \mathrm{H}_{2} \mathrm{O}\right.$ ) & 216 & $441^{*}$ & 140 & 97 \\
\hline \multicolumn{5}{|l|}{ Feeding Tests } \\
\hline 24 hr Food Deprivation & 118 & 97 & $128^{*}$ & 126 \\
\hline Amphetamine Treatment & $\dagger$ & $\dagger$ & $\dagger$ & $\dagger$ \\
\hline
\end{tabular}

Intakes of $\mathrm{OB}-1$ and $\mathrm{OB}-10$ are presented as a percentage of control intakes.

${ }^{*} p<0.05$ compared to control values

$\dagger$ Dose dependent suppression of food intake, $p>0.05$ compared to control.

days of age, $\mathrm{F}(16,464)=1.73$, posttest $p<0.05$. Male OB-10 rats weighed significantly more than male $O B-1$ rats from 40-150 days of age, $F(16,464)=1.73$, posttest $p<0.05$, whereas female OB-10 and OB-1 rats were not significantly different for body weight throughout development $(p>0.05)$. Body weights of OB-1 and OB-10 rats were not significantly different at 20 and 30 days of age $(p>0.05)$. Meaningful statistical comparisons between bulbectomized groups younger than 20 days of age are not possible because of the differences in recovery times from bulbectomies and the differences in the diets.

Ingestive measures. All daily water intakes and intakes on the specific drinking, taste, and feeding tests were corrected for body weight ( $\mathrm{ml}$ or $\mathrm{g}$ intake $/ 100 \mathrm{~g}$ body weight). Presented in Table 1 are the percentage of control intakes for male and female OB- 1 and OB-10 rats on drinking, taste, and feeding tests.

Daily drinking and drinking test intakes. No statistically significant differences were found for daily water intakes between bulbectomized and control groups from 30-150 days of age $(p>0.05)$. Bulbectomized rats were singificantly different from control rats on drinking tests for only 2 water intake comparisons (out of 12 possible comparisons). These differences were that OB-10 female rats were significantly hyperdipsic in response to cellular dehydration (injections of $16 \% \mathrm{NaCl}$ ) relative to control females, and $\mathrm{OB}-1$ females were significantly hypodipsic relative to control females following $24 \mathrm{hr}$ water deprivation $(p<0.05)$.

Taste tests. With the exception of OB-1 male rats (which responded at control levels on all taste tests), olfactory bulbectomy at 1 (females only) or 10 (males and females) days of age resulted in significantly reduced intakes of quinine adulterated water as compared to control rats $(p<0.05)$. Male and female OB-10 rats and female OB- 1 rats consumed at least $50 \%$ less of the $0.01 \%$ and $0.05 \%$ quinine solutions than did their respective controls. Although $\mathrm{OB}-1$ and $\mathrm{OB}-10$ males preferred $0.1 \% \mathrm{NaCl}$ solution over tap water $(p>0.05)$, only female $\mathrm{OB}-1$ rats preferred significantly more $\mathrm{NaCl}$ solution than control rats $(p<0.05)$. OB-10 female rats had similar relative intakes of $\mathrm{NaCl}$ solution to tap water compared to control females $(p>0.05)$ (Table 1$)$.

Feeding tests. The only rats to be significantly different from controls for food intakes following $24 \mathrm{hr}$ food deprivation were the $\mathrm{OB}-10$ males; they consumed significantly more than controls on this test $(p<0.05)$. All bulbectomized rats suppressed their food intakes as a function of increasing doses of D-amphetamine sulfate as did control rats $(p>0.05)$ (Table 1).

\section{DISCUSSION}

The present results show that a majority $(60 \%)$ of rats bulbectomized at 1 day of age (OB-1) survive through the preweaning period by suckling from the dam, and these results agree with recent reports [31]. In addition, we have shown that the OB-1 rats that survive the weaning period will survive through adulthood ( 150 days of age) by ingesting dry rat pellets and water. In contrast to previous reports [25], we found that all rats bulbectomized at 10 days of age (OB-10) and housed with the dam died within 6 days postsurgery. Thus, for OB-1 and OB-10 rats housed with the dam, OB-1 rats had a higher survival rate than $O B-10$ rats. The results of this comparison are in contrast to previous reports [25], and the discrepancy between studies may be related to differences in species of rat, surgical procedures, completeness of bilateral bulbectomy, housing temperature and so on ( e.g., [26]).

While OB-10 rats had absolute mortality rates when housed with the dam, they displayed an ability to early-wean to special diets and a $68 \%$ survival rate was achieved with this procedure. The survival rates of $\mathrm{OB}-1$ and early-weaned OB-10 rats were approximately equal $(60 \%$ and $68 \%$, respectively).

The postsurgical body weights of $\mathrm{OB}-10$ rats housed with the dam was a decreasing function until death at 6 days postsurgery. The OB-1 and early-weaned OB-10 rats displayed severe body weight reduction relative to controls in the early postsurgical period when they dropped to $40-60 \%$ of control body weights. This relative body weight reduction attenuated through 60-70 days of age when body weights of OB-1 and early-weaned OB-10 rats stabilized at approximately $70-80 \%$ of control body weights.

In response to specific feeding and drinking challenges administered at least 25 days postsurgery, the $O B-1$ and OB-10 rats displayed food and water intakes that generally approximated the intakes of control rats. However, most of the OB-1 and OB-10 rats displayed attenuated intakes of quinine adulterated water, relative to controls, and this may indicate an enhanced sensitivity to gustatory stimulation. The bulbectomized rats also tended to display enhanced preference for a saline solution over tap water relative to controls. These indications of altered gustatory sensitvities (which require more detailed study) are not unique to rats 
bulbectomized during infancy, as rats bulbectomized as adults also display similar responses $[10,12,19]$.

\section{EXPERIMENT 2}

Experiment 2 evaluated stomach contents of milk, nipple-attachment behaviors, and growth of rats sustaining olfactory bulbectomy at 1 or 10 days of age.

METHOD

\section{Animals}

The following groups of rats had appropriate surgical procedures as described in Experiment 1 . Twenty-four rats were bulbectomized at 1 day of age ( 12 males, 12 females), and 14 rats served as surgical controls ( 7 males, 7 females). Eighteen rats ( 10 males, 8 females) were bulbectomized at 10 days of age, and 10 rats ( 5 males, 5 females) served as surgical controls. Housing conditions were as described in Experiment 1. All pups were housed with littermates and their dam through sacrifice at 14 days of age (after completion of testing).

\section{Procedures}

Body weight was measured each day from the day of surgery through 14 days of age. Each day the pups' stomachs were visually inspected for the presence or absence of milk (stomach full, milk detectable, milk not detectable).

Nipple-attachment test. Two nipple-attachment tests were conducted the day following surgery and at 14 days of age, thus, 1-day-old bulbectomized pups were tested at 2 and 14 days of age and 10-day-old bulbectomized pups were tested at 11 and 14 days of age. It is realized that 1- and 10-day-old bulbectomized pups tested at 14 days of age have had different postoperative recovery times. These testing ages were chosen to enable identification of alterations in nipple-attachment behaviors soon after bulbectomy (the day following surgery) and at an older age immediately before eye opening ( 14 days of age). Pups were not deprived of their dam prior to testing when tested the day following surgery ( 2 or 11 days of age), but were deprived of their dam for $24 \mathrm{hrs}$ prior to testing at 14 days of age. This deprivation period was used because pilot research and others [15] have shown that control pups nipple-attach consistently on suckling tests at 2 and 11 days of age without deprivation, but a deprivation period is required for consistent nipple-attachment at 14 days of age. The dam in both tests was anesthetized with Nembutal (40 mg/kg body weight) and placed on her side on a heating pad with her ventrum facing towards the experimenter.

In the pup-on-nipple test (PON), the pup was held such that forelimb and head movements were not restricted as the snout was brought in contact with the nipple [15]. Latency to attach (sec) was measured from the time of initial contact with the nipple to attachment. Attachment was verified by gently pulling the pup from the dam. Some pups appeared to be attached but clearly were not when pulled from the dam; these pups were replaced to near their original position on the dam and the test was resumed. Pups failing to nippleattach within $5 \mathrm{~min}$ received an additional $5 \mathrm{~min}$ trial later in the session; therefore, the maximum latency to attach on this test was $10 \mathrm{~min}$. A $10 \mathrm{~min}$ latency was assigned to rats that failed to nipple-attach.

The pup-on-ventrum test (POV), unlike the PON test, required the pup to nipple-attach after independently locat-

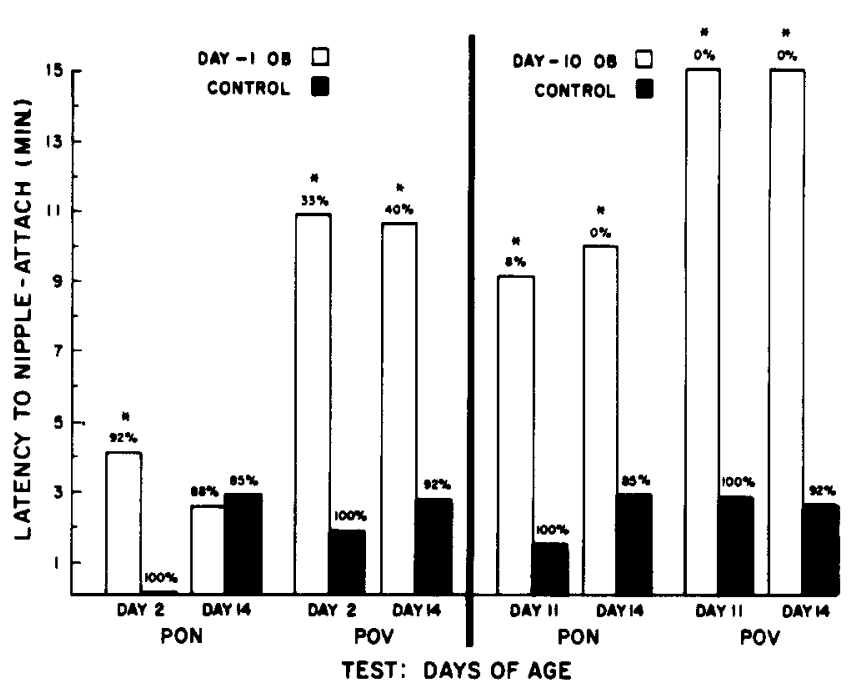

FIG. 3. Mean latencies-to-nipple-attach (in min) on PON and POV tests for control rats and rats bulbectomized at 1 (left) or 10 (right) days of age on the first day postsurgery ( 2 or 11 days of age) and at 14 days of age. Percentages above each bar represent the percentage of total pups within each of the respective groups nipple-attaching on the specific test. Asterisks denote significant differences in latencies of bulbectomized pups from those of control pups $(p<0.05)$. The maximum 10 (PON) or 15 (POV) minute latency was assigned to rats that failed to nipple-attach during the PON or POV tests.

ing the nipple [15]. Two littermates were placed with their snouts in contact with the dam's ventrum between the inguinal and pectoral sets of nipples. Latency to nipple-attach was measured as the time of initial contact with the ventrum to successful nipple-attachment. This was evidenced by a stereotypic behavior pattern of the pup kneading the nipple area followed temporarily by a lack of forelimb movement and sucking movements with the mouth on the nipple. Pups failing to nipple-attach within $15 \mathrm{~min}$ were scored as having a 15 min latency.

Pups with surgery at 1 day of age (bulbectomized and controls) were tested on only one type of suckling test (PON or POV) at 2 days of age to minimize the time away from their dam. Eight of 24 pups with surgery at 10 days of age and tested at 11 days of age were also tested on only one suckling test (PON or POV); the remaining 16 pups were tested on both tests (PON and POV). Statistical comparisons of latency-to-attach data were accomplished with two-tailed $t$-tests for independent samples, and comparisons of numbers of pups attaching on a test were accomplished with chi-square test for independence.

\section{Post-Mortem Examination}

Procedures were as described in Experiment 1. Only rats sustaining complete bilateral olfactory bulbectomy were included in the data analyses.

\section{RESULTS}

\section{Nipple-Attachment}

Presented in Fig. 3 are the results of the nipple- 
attachment tests administered to rat pups bulbectomized at 1 day of age (OB-1) or 10 days of age (OB-10). Plotted and noted in Figure 3 are latencies to nipple-attach and percentages of pups nipple-attaching on the PON (pup held with snout in contact with the nipple) and POV (pup placed with snout in contact with the dam's ventrum) tests conducted when the OB-1 pups were 2 and 14 days of age and when the OB-10 pups were 11 and 14 days of age.

When tested at 2 days of age, OB- $1(92 \%)$ and control $(100 \%)$ pups did not significantly differ for the proportion of pups nipple-attaching during the PON tests $(p>0.05)$. However, latencies to nipple-attach for the $\mathrm{OB}-1$ rats were significantly longer than latencies of the control rats on the PON test at 2 days of age $(p<0.05)$. OB- 1 and control rats did not significantly differ for the proportion of pups nippleattaching or for latencies to nipple-attach on the PON test administered at 14 days of age $(p>0.05)$. The pups were required to independently locate the nipple for nippleattachment on the POV tests administered at 2 and 14 days of age, and the $O B-1$ rats displayed significantly smaller proportions of pups nipple-attaching on both days $(p<0.05)$, and the $\mathrm{OB}-1$ rats had significantly longer latencies to nippleattach on both days $(p<0.05)$.

On the PON tests administered at 11 and 14 days of age, the proportion of $O B-10$ rats nipple-attaching was significantly smaller than the proportion for control rats $(p<0.05)$, and the OB-10 rats had significantly elongated latencies to nipple-attach as compared to control rats $(p<0.05)$. The OB-10 group also had significantly fewer pups nippleattaching $(p<0.05)$ and longer latencies $(p<0.05)$ as com pared to control rats on the POV tests conducted when the rats were 11 and 14 days of age.

\section{Survival and Growth}

There were no gender differences for control, $\mathrm{OB}-1$, or OB-10 rats on measures of survival, presence of stomach milk, or body weight at the ages examined $(p>0.05)$.

Visual inspection of the pups revealed that most $(83 \%)$ of the OB-1 rats had milk in their stomachs within $48 \mathrm{hrs}$ postsurgery. While $100 \%$ of the OB-1 rats were alive at 4 days postsurgery, $40 \%$ of the OB-1 pups died by 14 days of age ( 13 days postsurgery). There were no significant differences between the proportions of OB-1 rats that survived nipple-attachment tests administered at 2 days of age versus 14 days of age $(p>0.05)$. Thus, less than half of the rats bulbectomized at 1 day of age had died by 14 days of age, and the early nipple-attachment tests (administered at 2 days of age) did not affect mortality. Body weights of $O B-1$ rats at 4 days of age $(\mathrm{OB}-1=9.4 \mathrm{~g}$, control $=15.2 \mathrm{~g})$ and at 14 days of age $(\mathrm{OB}-\mathrm{l}=17.1 \mathrm{~g}$, control $=36.2 \mathrm{~g})$ were significantly below body weights of control rats $(p<0.01)$.

None of the OB-10 rats had detectable milk in their stomachs from the first day postsurgery through sacrifice at 14 days of age. Eighty-seven percent of the $\mathrm{OB}-10$ rats survived through 14 days of age ( 4 days postsurgery), and the OB-10 survivors had body weights which were $45 \%$ below control body weights at 14 days of age $(\mathrm{OB}-10=20.4 \mathrm{~g}$, control $=37.9 \mathrm{~g})(p<0.05)$. There were no significant differences in proportions of $\mathrm{OB}-10$ rats that survived nipple-attachment testing at 11 days of age, 14 days of age, or 11 and 14 days of age $(p>0.05)$.

\section{DISCUSSION}

The present results replicate and extend previous re- search on the effects of olfactory bulbectomy sustained by preweaning rats on nipple-attachment behaviors during $P O N$ and $\mathrm{POV}$ forms of testing in spite of a variety of experimental differences between studies, e.g., species of rat, surgical procedures, temperature of housing and testing environments, ages of rats at testing [27.28,31]. The present study showed that almost all of the rat pups bulbectomized at 1 day of age nipple-attached when held in contact with the nipple during PON tests at 2 days of age (92\% nipple-attached) or 14 days of age ( $88 \%$ nipple-attached). When required to independently locate the nipple for attachment during the $\mathrm{POV}$ tests, $30-40 \%$ of the neonatally bulbectomized pups successfully nipple-attached during tests at 2 and 14 days of age. In contrast, none $(0 \%)$ of the pups bulbectomized at 10 days of age nipple-attached during the POV tests administered at 11 or 14 days of age, and when held with their snouts on the nipple during the PON tests, only $8 \%$ of the pups nippleattached at 11 days of age and none $(0 \%)$ nipple-attached at 14 days of age.

In comparison to rat pups bulbectomized at 10 days of age, a higher proportion of pups bulbectomized at 1 day of age had milk in their stomachs by 48 hrs postsurgery, and a higher proportion of neonatally bulbectomized pups nipple-attached druing PON and POV tests. These results suggest that nipple-attachment behaviors using an anesthetized dam was an accurate predictor of survival for rats bulbectomized at 10 days of age. Almost none of these rats nipple-attached on PON or POV tests, none displayed milk in their stomachs, and, as shown in Experiment $\mathrm{I}$. none survived beyond 6 days postsurgery. Nipple-attachment behaviors were not as good a predictor of survival for $\mathrm{OB}-1$ rats. Almost all OB-1 rats nipple attached on PON tests. $30-40 \%$ nipple-attached on POV tests, $83 \%$ had milk in their stomachs within a few days postsurgery; yet, $60 \%$ survived as shown in Experiments 1 and 2. The best predictor of survival for OB-1 rats was their performance on the POV test. i.e., their ability to independently locate the nipple.

\section{GENERAL DISCUSSION}

Experiment 2 showed that rat pups bulbectomized at 1 day of age displayed less disruption of nipple-attachment behaviors (as evidenced by the PON and POV tests) and suckling from the dam (as evidenced by the presence of milk in their stomachs) than was found for pups bulbectomized at 10 days of age. Experiment 1 showed that $60 \%$ of the pups bulbectomized at 1 day of age survived through the age of weaning by suckling from the dam, while pups bulbectomized at 10 days of age (and kept with the dam) all (100\%) died by 6 days postsurgery. However, a $68 \%$ survival rate for 10-day-old bulbectomized rats was achieved when the rats were removed from the dam and given access to special diets, i.e., an early weaning procedure. Experiment 1 also showed that 1-day-old bulbectomized rats and early-weaned 10-day-old bulbectomized rats were $20-30 \%$ below control body weights through 150 days of age, yet, bulbectomized rats did not display any severe or consistent deficits in feeding or drinking behaviors later in development. However, bulbectomized rats tended to display altered responses on taste tests administered during development and as adults.

The present demonstration of higher survival rates of pups bulbectomized at 1 versus 10 days of age is at odds with some $[25,27,28]$, but not all [31], of the research literature. The discrepancy across studies may be related to a number 
of factors including strain of rat, surgical efficiency and procedures, completeness of bulbectomy, temperature of housing conditions, and so on (e.g., [26]). Nevertheless, the present results and those of others [31] conclusively demonstrate that a majority of rat pups bulbectomized within the first few days of life are capable of sustaining growth by nippleattaching and suckling from the dam. Under these conditions, the pups most likely are utilizing tactile and gustatory cues for maintaining suckling behaviors $[14,16,18]$. However, documented postnatal neurogenesis and/or regeneration of olfactory tissue $[6,13,17]$ suggests the possibility of functional recovery of olfaction to some degree.
Thus, the present study indicates that neonatal rats may be capable of using sensory cues, other than olfaction, to maintain suckling behaviors when the olfactory bulbs are removed during the first few days of life. This does not seem to be the case for older rats (e.g., 10 days of age) sustaining olfactory bulbectomy at an age when olfactory cues would be more salient, and the relationship between olfaction and the nipple as a food source has been established. These results, along with other research (e.g., [2,5]), further document the behavioral plasticity of the neonatal rat following damage to neural substrates important for the regulation of ingestive behaviors.

\section{REFERENCES}

1. Alberts, J. R. Producing and interpreting experimental olfactory deficits. Physiol. Behav. 12: 657-670, 1974.

2. Almli, C. R. The ontogeny of feeding and drinking: Effects of early brain damage. Neurosci. Biobehav. Rev. 2: 281-300, 1978.

3. Almli, C. R. and R. S. Fisher. Infant rats: Sensorimotor ontogeny and effects of substantia nigra destruction. Brain Res. Bull. 2: 425-459, 1977.

4. Almli, C. R. and G. T. Golden. Preweanling rats: Recovery from lateral hypothalamic damage. J. comp. physiol. Psychol. 90: $1063-1074,1976$.

5. Almli, C. R., D. L. Hill, N. T. McMullen and R. S. Fisher Newborn rats: Lateral hypothalamic damage and consummatory-sensorimotor ontogeny. Physiol. Behav, 22: 767-773, 1979.

6. Altman, J. Autoradiographic and histological studies of postnatal neurogenesis IV. Cell proliferation and migration in anterior forebrain, with special reference to persisting neurogenesis in the olfactory bulb. J. comp. Neurol. 137: 443$458,1969$.

7. Blass, E. M. and M. H. Teicher. Suckling. Science 210: 15-22, 1980

8. Blass, E. M., M. H. Teicher, C. P. Cramer, J. P. Bruno and W. G. Hall. Olfactory, thermal and tactile controls of suckling in preaudial and previsual rats. J. comp. physiol. Psychol. 91: 1248-1266, 1977.

9. Bruno, J. P., M. H. Teicher and E. M. Blass. Sensory determinants of suckling behavior in weanling rats. J. comp. Physiol. 94: $115-127,1980$.

10. Chiarviglio, E. Effects of lesions in the septal area and olfactory bulbs on sodium chloride intake. Physiol. Behav. 4: 693-697, 1969.

11. Cicchetti, D. V. Extension of multiple-range tests to interaction tables in the analysis of variance: A rapid approximate solution. Psychol. Bull. 77: 405-408, 1972.

12. Gessell, C. and G. L. Fisher. Caffine aversion and saccharine preference in rats without olfactory bulbs. Physiol. Behav. 3: 523-525, 1968

13. Graziadei, P. P. C., R. R. Levine and G. A. Monti Graziadei. Regeneration of olfactory axons and synapse formation in the forebrain after bulbectomy. Proc. natn. Acad. Sci. U.S.A. 75: $5230-5234,1978$.

14. Hall, W. G. and T. E. Bryan. The ontogeny of feeding in rats: II. Independent ingestive behavior. J. comp. physiol. Psychol. 94: $746-756,1980$

15. Hall, W. G., C. P. Cramer and E. M. Blass. Ontogeny of suckling in rats: Transitions toward adult ingestion.J. comp. physiol. Psychol. 91: 1141-1155, 1977.
16. Hill, D. L. and C. R. Almli. Ontogeny of chorda tympani nerve responses to gustatory stimuli in the rat. Brain Res. 197: 27-38, 1980.

17. Hinds, J. W. Autoradiographic study of histogenesis in mouse olfactory bulb. II. Cell proliferation and migration. J. comp. Neurol. 134: 305-322, 1968.

18. Hofer, M., A. Fisher and H. Shair. Snout denervation disrupts suckling in young rats. Paper presented at the meeting of the International Society for Developmental Psychobiology, Atlanta, GA, 1979.

19. Kahn, M. and E. Stellar. Alcohol preference in normal and anosmic rats. J. comp. physiol. Psychol. 53: 571-575, 1960.

20. Kling, A. Effects of rhinencephalic lesions on endocrine and somatic development in the rat. Am. J. Physiol. 206: 1395-1400, 1964.

21. Larue, C. G. and J. LeMagnen. Olfactory control of meal pattern in rats. Physiol. Behav. 9: 817-821, 1972.

22. McMullen, N. T. and C. R. Almli. Septal destruction in infant rats and the ontogeny of drinking behaviors. Brain Res. Bull. 4: 373-380, 1979.

23. Pollack, E. I. and B. D. Sachs. Male copulatory behavior and female maternal behavior in neonatally bulbectomized rats. Physiol. Behav. 14: 337-343, 1975.

24. Richter, C. P. Increased salt appetite in adrenalectomized rats. Am J. Physiol. 115: 115-161, 1936.

25. Rouger, Y., E. Tobach and T. C. Schneirla. Development of olfactory function in the rat pup. Am. Zool. 7: 792-793, 1967.

26. Sachs, B. D. and A. R. Lumia. Comment: Is stress due to shipment of animals a confounding variable in developmental research? Devl Psychobiol. 14: 1-3, 1981.

27. Singh, P. J. and E. Tobach. Olfactory bulbectomy and nursing behavior in rat pups (Wistar DAB). Devl. Psychobiol. 8: 151$164,1975$.

28. Singh, P. J., A. M. Tucker and M. A. Hofer. Effects of nasal ZnS04 irrigation and olfactory bulbectomy on rat pups. Physiol. Behav. 17: 373-382, 1976.

29. Teicher, M. H. and E. M. Blass. Suckling in newborn rats: Eliminated by nipple lavage, reinstated by pup saliva. Science 193: 422-425, 1976

30. Teicher, M. H. and E. M. Blass. First suckling response of the newborn albino rat: The roles of olfaction and amniotic fluid. Science 198: 635-636, 1977.

31. Teicher, M. H., L. E. Flaum, M. Williams, S. J. Eckhert and A. R. Lumia. Survival, growth and suckling behavior of neonatally bulbectomized rats. Physiol. Behav. 21: 553-561, 1978.

32. Tukey, J. W. Comparing individual means in the analysis of variance. Biometrics 5: 99-114, 1949.

33. Zucker, I. Light-dark rhythms in rat eating and drinking behavior. Physiol. Behav. 6: 114-126, 1971. 